\title{
STATISTICAL COMPARISON OF CLEAN AND MEM
}

\section{ISHIGURO}

The Institute of Statistical Mathematics, 6-7 Minami-Azabu 4-chome, Minato-ku, Tokyo 106 Japan

\section{ISHIGURO AND K.-I. MORITA}

Nobeyama Radio Observatory, National Astronomical Observatory, Nobeyama, Minamimaki, Minamisaku, Nagano 384-13 Japan

\section{Y. MURATA}

The Institute of Space and Astronomical Science, 1-1 Yoshinodai 3-chome, Sagamihara 229 Japan

\section{INTRODUCTION}

Assume that a set of visibility data

$$
\left\{\left(u_{j}, v_{j}, z_{j}\right) \mid j=1,2, \ldots, N\right\}
$$

is given where visibility $z_{j}$ observed at time $j$ can be expressed in terms of "true visibility" defined by the brightness distribution $T(x, y)$ and observation noise $\varepsilon_{j}$ :

$$
\begin{aligned}
& z_{j}=\tilde{T}\left(u_{j}, v_{j}\right)+\varepsilon_{j} \quad(j=1,2, \ldots, N) \\
& \tilde{T}(u, v)=\iint \exp \{2 \pi i(x u+y v)\} T(x, y) d x d y,
\end{aligned}
$$

where $\left\{\left(u_{j}, v_{j}\right)\right\}$ is a given set of points in the $u-v$ plane. It is assumed that $u-v$ plane is gridded with the interval $\Delta$. The dirty map obtained by

$$
M_{D}(x, y)=\Delta^{2} \sum_{j=1}^{N} \exp \left\{-2 \pi i\left(x u_{j}+y v_{j}\right)\right\} z_{j}
$$

is a crude estimate of the true brightness distribution. CLEAN and MEM are two typical methods to get better estimates.

Ishiguro $^{2}$ and Morita (1991) showed that the number of CLEAN components can be determined by the minimum WIC procedure. The purpose of the present paper is to show that WIC can be used to compare the performances of CLEAN and MEM.

\section{SIMULATION STUDY}

Dr. T.J. Cornwell's MEM routine "VM" and a CLEAN procedure were applied to data sets generated by assuming the true distribution shown in Figure I. 
Values of the minus twice of expected log likelihood ( -2 ELL ) and WIC for each obtained maps were calculated. Mean values of results of a hudred simulation runs are summarized in Table $I$.

Inspecting the table two facts are readily seen:

1. Since smaller value of "-2 ELL" implies better fit of the map (Akaike 1973), CLEAN procedure is judged to give better results for the present case. CLEAN map with 100 components is the best among the candidates.

2. It is also apparent from the table that "-2 ELL" values are successfully estimated by WIC values which are calculated not knowing the true brightness distribution $T(x, y)$.

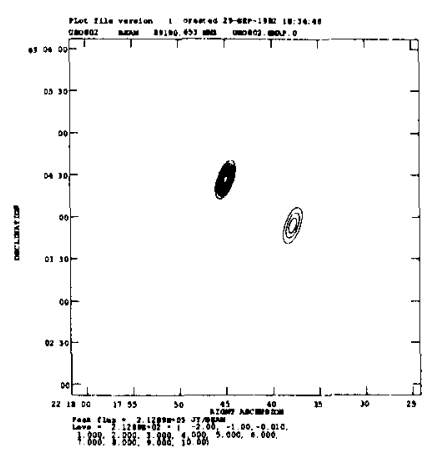

FIGURE I The true distribution $T(x, y)$ with two Gaussian components

TABLE I Information-Criterion

\begin{tabular}{lcc}
\multicolumn{1}{c}{ Map } & -2 ELL & WIC \\
\hline MEM Map(small SIGMA) & 21466.94 & 21527.90 \\
MEM Map(medium SIGMA) & 20556.41 & 20632.21 \\
MEM Map(large SIGMA) & 21357.34 & 21390.80 \\
CLEAN Map(N=100) & 20305.85 & 20400.87 \\
CLEAN Map(N=200) & 20317.31 & 20422.90 \\
True Model & $20276.32^{b}$ & 20324.91
\end{tabular}

${ }^{a}$ Best map

${ }^{b}$ Theoretical lower bound of -2 ELL value

\section{REFERENCES}

Akaike, H. 1973, Information theory and an extension of the maximum likelihood principle, 2nd International Symposium on Information Theory (Petrov, B. N. and Csaki, F. eds.), Akademiai Kiado, Budapest, pp.267281. (Reproduced in Breakthroughs in Statistics, volume 1, S. Kotz and N. L. Johnson, eds., Springer Verlag, New York, (1992).)

(Ishiguro, M.) ${ }^{2}$ and Morita, K.-I. 1991, Application of an Estimator-Free Information Criterion(WIC) to Aperture Synthsis Imaging, in Radio Interferometry: Theory, Techniques and Applications (Cornwell, T.J. and Perley, R.A. eds.), IAU Coll. 131, ASP Conference Series, 19, 243. 